\title{
Hirschsprung Disease in Adults: A Case Report in a Tertiary Hospital in West Africa
}

\author{
Ananivi Sogan ,(MD, Surgeon, MSc in Anatomy) \\ Efoe-Ga Amouzou, (MD, Surgeon) \\ Yawa Sesime Sanni, (MD, Pediatric Surgeon) \\ Hassiatou Sabi Couscous, (MD, Radiologist) \\ Victor Adjenou, (MD, Radiologist, Professor) \\ Komlanvi James, (MD, Surgeon, Professor in Anatomy) \\ University of Lomé, Togo
}

Doi:10.19044/esj.2022.v18n8p208

Submitted: 19 September 2021

Copyright 2022 Author(s)

Accepted: 16 February 2022

Published: 28 February 2022

Under Creative Commons BY-NC-ND

4.0 OPEN ACCESS

Cite As:

Sogan A., Amouzou E., Sanni Y.S., Couscous H.S., Adjenou V., \& James K.,(2022). Hirschsprung Disease in Adults: A Case Report in a Tertiary Hospital in West Africa European Scientific Journal, ESJ, 18 (8), 208.

https://doi.org/10.19044/esj.2022.v18n8p208

\begin{abstract}
Hirschsprung disease in adults is a rare and frequently misdiagnosed cause of long-standing and refractory constipation. Surgical procedures initially developed for pediatric patients are applied to adults with varying degrees of success. A nineteen-year-old girl was diagnosed with Hirschsprung disease in a tertiary hospital of low technology level in Togo. Soave-Boley's procedure was used as definitive surgical management which resulted in a good outcome.
\end{abstract}

Keywords: Hirschsprung disease, adult, surgery, Togo

\section{Introduction}

Hirschsprung disease is a rare congenital condition in which an absence of ganglion cells in the distal colon results in functional obstruction, leading to a long-standing and refractory constipation and a megacolon (Natsikas \& Sbarounis, 1987). In rare cases, the condition remains undiagnosed until adolescence or adult age because hypertrophy of the active normally innervated proximal bowel compensates for the prolonged partial recto-sigmoid obstruction (Natsikas \& Sbarounis, 1987; Todd, 1977). In 
addition, use of enemas and cathartics to manage constipation may succeed in masking the condition until acute occlusion of the proximal colon, fecal impaction, or megacolon involves urgent surgical intervention (Vorobyov et al., 2010). Since the discovery of Hirschsprung disease, many surgical techniques have been performed, including laparoscopic procedure (Jarry \& Faucheron, 2011). According to the conditions of tertiary hospitals, SoaveBoley's technique has been applied in reported cases of Hirschsprung disease in adults.

\section{Patient And Observation}

A 19-year-old girl was admitted and diagnosed with acute intestinal occlusion. She experienced painful abdominal distension with a mass and also vomiting. More so, the patient had a history of chronic constipation and often eased herself with daily enemas since childhood. Interestingly, she had never been diagnosed due to ignorance and poverty of her family. Clinically, this patient presented normal blood pressure $(100 / 70 \mathrm{mmHg})$, acceptable general status, important abdominal distension without intestinal perforation signs, and empty rectal ampoule. Standard laboratory investigation was normal. Abdominal and pelvis CT scan revealed a colonic obstruction due to a huge fecal impaction around the whole sigmoid colon and distal left colon. This part of the bowel was so excessively dilated that it almost occupied the totality of the abdomen. Figure 1 and 2 shows dilated colon (rectum seemed narrow, empty with normal diameter). The probability of Hirschsprung disease of the recto-sigmoid junction was raised and, 24 hours later, a left transversal colostomy was performed to relieve symptoms. During the surgical process, through a median laparotomy, a huge bowel (sigmoid colon) of $35 \mathrm{~cm} \times 25$ $\mathrm{cm}$ was seen and $10 \mathrm{~kg}$ of faeces was evacuated. Thereafter, the rectosigmoid resection was done (Figure 3, 4). Hartmann procedure which extended to the splenic flexure was performed. The diagnosis of Hirschsprung disease was confirmed on a histological examination of resected specimen. It was a short form of aganglionosis including recto-sigmoid junction. Three months later, Soave-Boley's procedure was performed for definitive surgery. Postoperative recovery (18 months after definitive surgery) was uneventful and the patient described complete resolution of symptoms with normal continence. Bowel movement was once or twice a day with normal stool.

\section{Comments}

Diagnosis of Hirschsprung disease in adults is carried out if a patient with colorectal aganglionosis is older than the arbitrary age of 10 years at the time of diagnosis (Duncan et al., 2009). The real incidence of this disease is unknown due to probable under-diagnosis in the adult population (Chen et al., 2006). While a vast majority of patients with Hirschsprung disease are 
diagnosed in the neonatal period, Hirschsprung himself acknowledged this was a disease of all ages (Lesser et al., 1979). In theory, undiagnosed adult patients who are able to live with this congenital abnormality for so many years have more mild symptoms. This is due to a compensatory contribution of the distal and proximal colon to the aganglionic segment (Schlund et al., 2020). Most of these patients have a short or an ultra-short segment of aganglionosis (Duncan et al., 2009). While the symptoms may be mild and can be treated with cathartic agents, the dilated proximal colonic segment is at risk of decompensation. This could potentially result in an emergent situation such as an acute obstruction or perforation (Schlund et al., 2020). The reported case was an acute bowel obstruction in a 19-year-old girl with a history of constipation and abdominal discomfort.

Standard preoperative investigations include a barium enema or CT scan (Jarry \& Faucheron, 2011). However, abdominal and pelvis CT scan was used in this case as recommended by some authors for acute abdominal syndrome (Franchi-Abella \& Pariente, 2008). Nonetheless, confirmation of the diagnosis is necessary before definitive surgery.

Traditionally, four surgical procedures are employed to treat Hirschsprung disease in adults: the Lynn's, Soave-Boley's, the Swenson's, and the Duhamel's procedures (Adamou et al., 2019; Kuroda, 2019). These procedures are also used for classic Hirschsprung disease in infancy. Although all procedures involve the resection of histologically confirmed aganglionic bowel, they differ in the mode of reconstruction and outcomes (Duncan et al., 2009). Major complications were found with Soave-Boley's procedure, Swenson's procedure, and Duhamel's procedure at 19.3\%, 28.6\%, and 7.4\%, respectively. This indicated poor functional results, respectively, at $12 \%$, 13.8\%, and $2.4 \%$ (Jarry \& Faucheron, 2011; Nasayuki et al., 2005). Nowadays, new approaches are emerging in the surgical treatment of Hirschsprung disease in adults. Laparoscopic recto-sigmoid resection with a transanal colonic pull-through, which is followed by delayed colo-anal anastomosis, represents a valid alternative in the treatment of Hirschsprung disease in adult patients. This is because it involves less invasive surgery, does not require a preventive diverting stoma, and shows anastomotic security (Jarry \& Faucheron, 2011). In a nutshell, laparoscopic approaches provide fewer complications. Unfortunately, it is not practiced in tertiary hospitals in Togo since there is no adequate equipment for laparoscopic procedure. This is why Soave-Boley's procedure is more widely used. However, eighteen months after the procedure, the patient does not complain about any complications. In Niger, Adamou et al. (2019) also used Soave-Boley's technique and had a good result in their case report. In Nigeria, authors did not use laparoscopic approaches to combat Hirschsprung disease in adults, but 
they practised Swenson and Lynn's procedure which produced satisfactory outcome (Arowolo et al., 2013)

\section{Conclusion}

Due to ganglion cell absence in distal colon, Hirschsprung disease is largely known in childhood. Although this condition is undiagnosed in the early stages of childhood, it is found in adults after a long period of constipation. This is observed in the reported case of a patient who was admitted for a colonic obstruction by a huge fecal impaction. However, this condition was managed by a two-step surgery which led to good outcomes.

\section{Appendices}

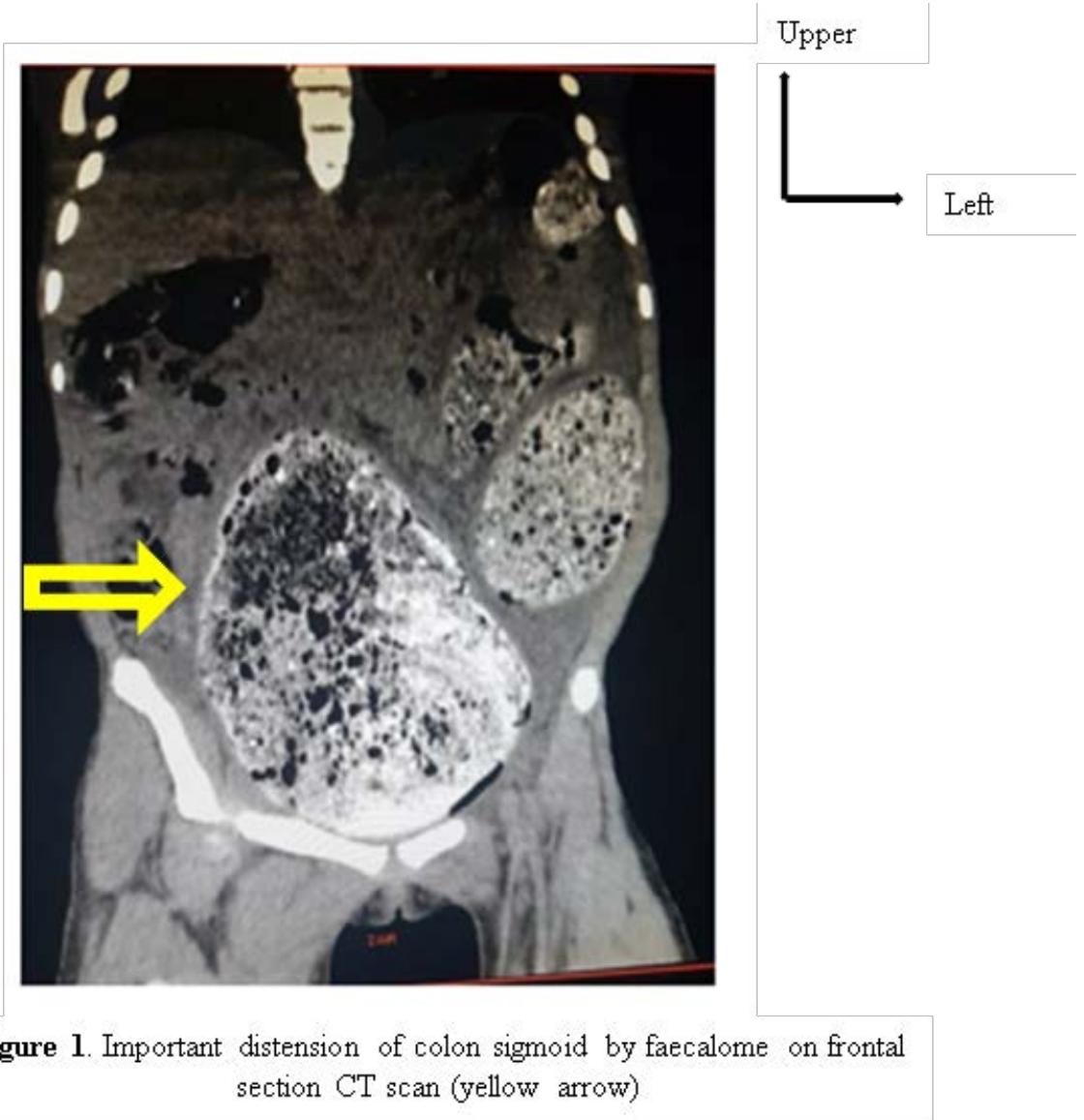



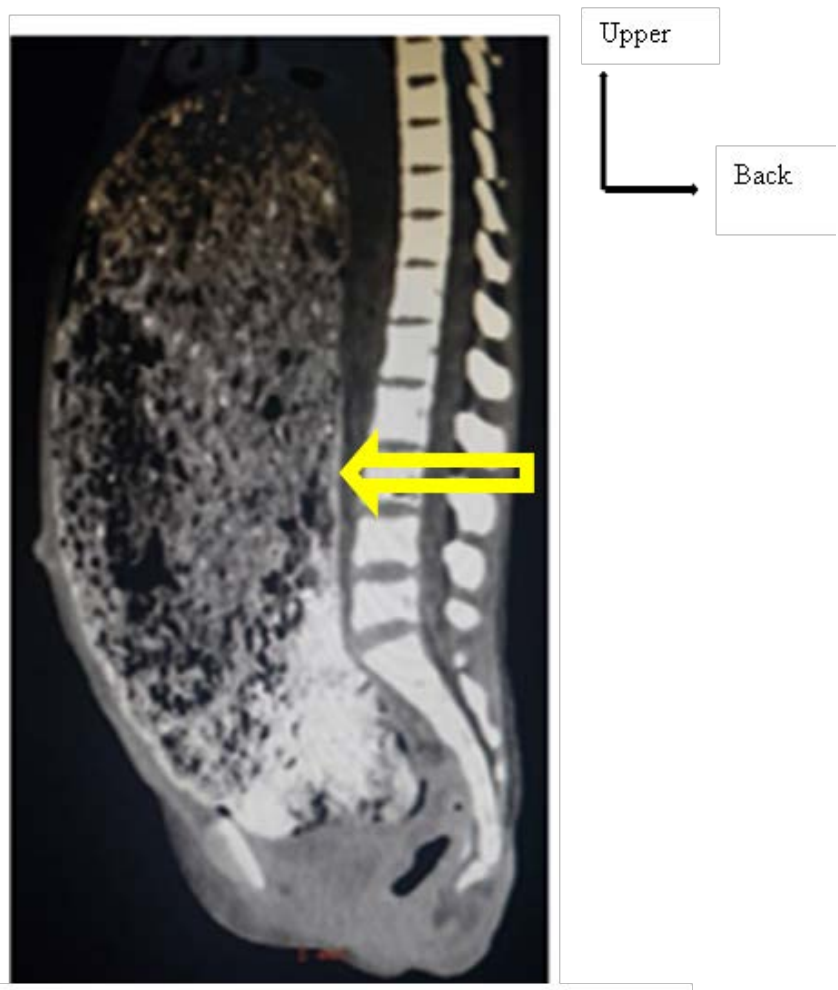

Figure 2. Important distension of colon sigmoid by faecalome on sagittal section CT scan (yellow arrow)

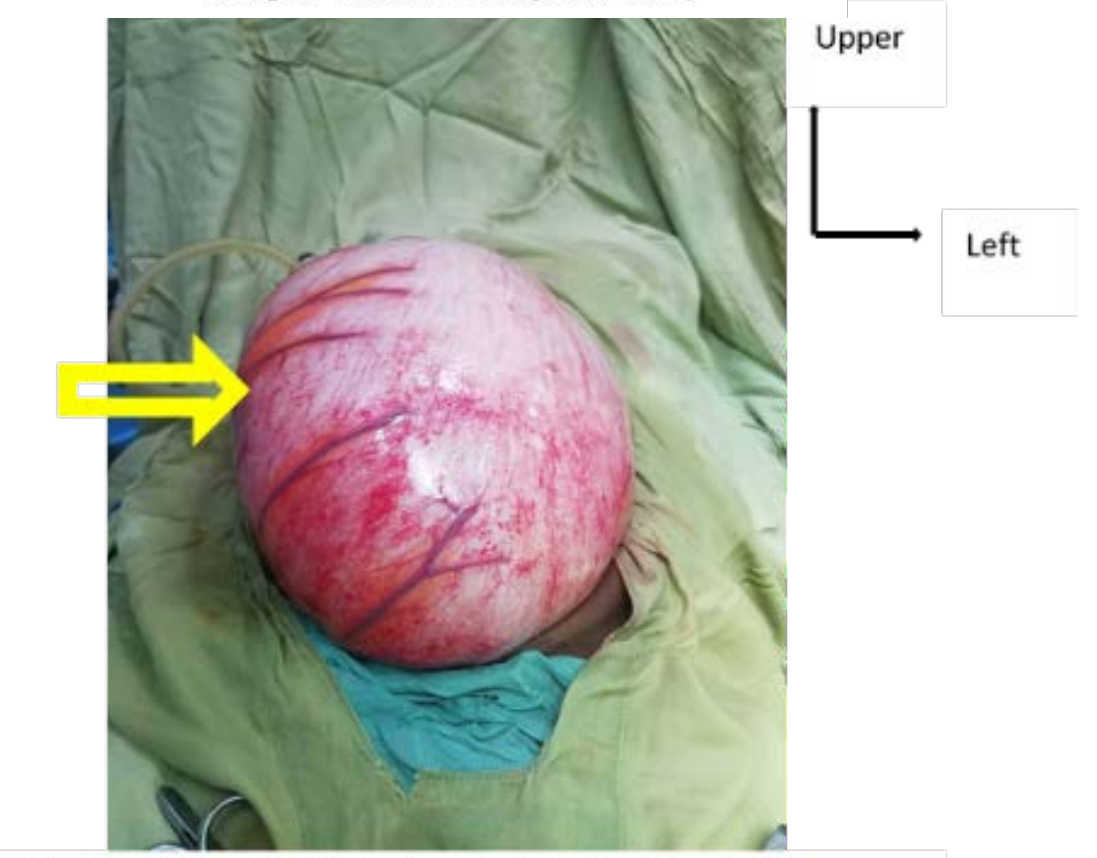

Figure 3: Important distension of sigmoid colon (yellow arrow) by fecal impaction, during laparotomy. 


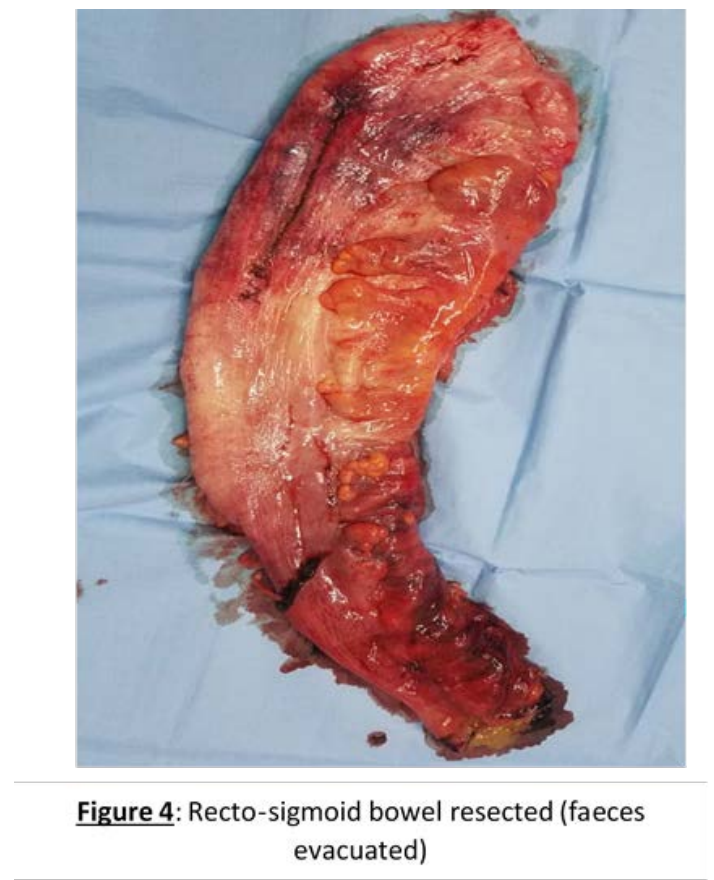

\section{References:}

1. Adamou, H., Magagi, I., Habou, O., Adakal, O., Aboulaye, M., \& Robnodji, A. (2019). Diagnosis and surgical approach of adult Hirschsprung disease: About two observations and review of the literature. Case series, Annals of Medicine and Surgery, 48, 59-64.

2. Arowolo, OA., Lawal, OO., Adisa, AO., Adetiloye, VA., Afolabi, AI., \& Sowande, OA. (2013). Adulthood Hirschsprung disease: a report of 04 cases in Ile-Ife, Nigeria. Afr J Med Med Sci, 42(3), 277-82.

3. Chen, F., Winston, J., Jain, S., \& Frankel, W. (2006). Hirschsprung disease in a young adult: report of a case and review of the literature. Ann Diagn Pathol, 10(6), 347-351.

4. Duncan, ND., Plummer, J., Dundas, SE., Martin, A., \& McDonald, AH. (2009). Adult Hirschsprung disease in Jamaïca: operative treatment and outcome. Colorectal Disease, 13, 454-458.

5. Franchi-Abella, S. \& Pariente, D. (2008). Le radiologue non spécialiste face au scanner pédiatrique: les indications du scanner abdominal et les protocoles. Journal de Radiologie, 89(10), 1384-1385.

6. Jarry, J. \& Faucheron, JL. (2011). Laparoscopic rectosigmoid resection with transanal colonic pull-through and delayed colo-anal anastomosis: A new approach to adult Hirschsprung disease. Diseases of the colon \& rectum, 54(10): 1313 - 1319. 
7. Kuroda, T. (2019). Soave-Denda-Boley Procedure. In: Taguchi T., Matsufuji H., Ieiri S. (eds) Hirschsprung Disease and the Allied Disorders. Springer, Singapore. Doi: 10.1007/978-981-13-3606-5_21

8. Lesser, P., El-Nahas, A., Lukl, P., Andrews, P., Schuler, J., \& Filtzer, H. (1979). Adult - onset Hirschsprung disease. JAMA, 242(8), 747748.

9. Nasayuki, M., Kaku, E., Shotaro, M., Keiichi, O., Noritake, T., \& Eiji, U. (2005). Hirschsprung disease in adults: report of case and review of the literature. J Nippon Med Scch, 72(2), 113 - 120.

10. Natsikas, NB. \& Sbarounis, CN. (1987). Adult Hirschsprung disease: an experience with the Duhamel-Martin procedure with special reference to obstructed patients. Dis Colon Rectum, 30, 204-206.

11. Schlund, D., Jochum, SB., Favuzza, J., Hayden, DM., Pillai, SB., \& Saclarides, TJ. (2020).A national analysis of operative treatment of adult patients with Hirschsprung disease. International Journal of Colorectal Disease, 35, 169-172.

12. Todd, IP. (1977). Adult Hirschsprung disease. Br J Surg, 64, 311-312.

13. Vorobyov, GI., Achkasov, SI., \& Biryukov, OM. (2010). Clinical features' diagnostics and treatment of Hirschsprung disease in adults. Colorectal Dis, 12, 1242-1248. 